\title{
Implication of species change of Nontuberculous Mycobacteria during or after treatment
}

Jong Sik Lee', Jong Hyuk Lee², Soon Ho Yoon², Taek Soo Kim³, Moon-Woo Seong ${ }^{3}$, Sung Koo Han and Jae-Joon Yim $^{1 *}$

\begin{abstract}
Background: Co-existence or subsequent isolation of multiple nontuberculous mycobacteria (NTM) species in same patient has been reported. However, clinical significance of these observations is unclear. The aim of this study was to determine clinical implications of changes of NTM species during or after treatment in patients with NTM lung disease.

Methods: Patients with NTM lung disease, who experienced changes of NTM species during treatment or within 2 years of treatment completion between January 1, 2009 and December 31, 2015, were included in the analysis. Demographic, clinical, microbiological, and radiographic data were reviewed and analyzed.

Results: During the study period, 473 patients were newly diagnosed with NTM lung disease. Treatment was started in 164 patients (34.6\%). Among these 164 patients, 16 experienced changes of NTM species during or within 2 years of treatment completion. Seven showed changes from M. avium complex (MAC) to M. abscessus subspecies abscessus (MAA) and five patients displayed changes from M. abscessus subspecies massiliense (MAM) to MAC. With isolation of new NTM species, 6 out of 7 patients with change from MAC to MAA reported worsening of symptoms, whereas none of the five patients with change from MAM to MAC reported worsening of symptoms. All MAA isolated during or after treatment for MAC lung diseases showed inducible resistance to clarithromycin.
\end{abstract}

Conclusions: Change of NTM species may occur during or after treatment for NTM lung disease. Especially, changes from MAC to MAA is accompanied by symptomatic and radiographic worsening as well as inducible resistance to clarithromycin.

Keywords: Nontuberculous mycobacteria species change, Mycobacterium avium Complex, Mycobacterium abscessus subspecies abscessus, Clarithromcycin resistance

\section{Background}

Nontuberculous mycobacteria (NTM) are ubiquitous in environments including natural or treated water and soil. They have a relatively low pathogenicity but can cause lung disease in immunocompetent as well as immunocompromised hosts. [1, 2] Currently, approximately 150 NTM species have been identified. $[3,4]$ The

\footnotetext{
* Correspondence: yimjj@snu.ac.kr

${ }^{1}$ Division of Pulmonary and Critical Care Medicine, Department of Internal Medicine, Seoul National University College of Medicine, 101, Daehak-ro, Jongno-gu, Seoul 03080, Republic of Korea

Full list of author information is available at the end of the article
}

incidence and prevalence of NTM lung disease are rising worldwide. $[1,5]$ The distribution of causative species of NTM lung disease varies according to country and region. In South Korea, M. avium complex (MAC) is the most common, comprising approximately $60 \%-70 \%$ of all cases, followed by $M$. abscessus complex, comprising approximately $20 \%-30 \%$. [6-9].

Isolation of NTM different from initial NTM species in same patients has been reported. Co-culture of MAC was reported among $20 \%$ of patients with $M$. abscessus subspecies abscessus (MAA). [10] We also reported wide spectrum of NTM species changes including change of 

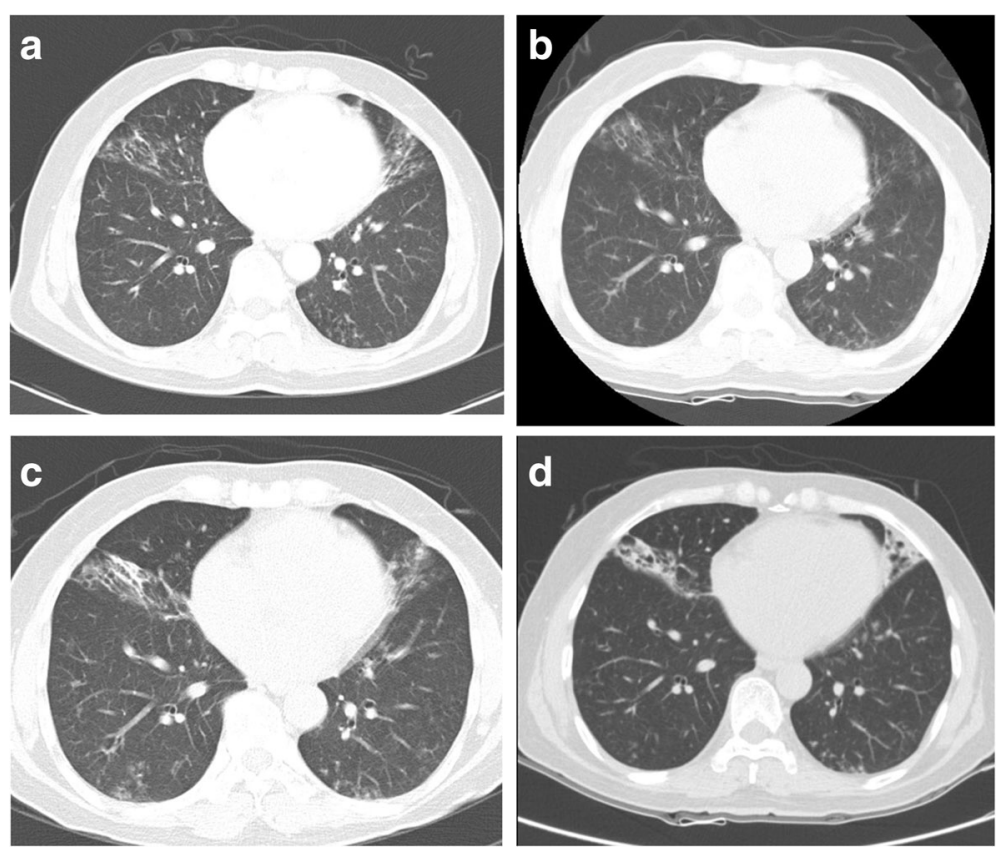

Fig. 1 a Chest CT scan of a patient with symptomatic Mycobacterium intracellulare lung disease before initiation of treatment. The scan shows bronchiectasis, nodules, and reticular densities in the right middle lobe and lingular segment. Treatment started 4 weeks after checking this $C T$ scan. b Chest CT scan at 6 months after the initiation of treatment showing substantial improvement. c Chest CT scan at 12 months after the completion of 18-month treatment for $M$. intracellulare lung disease. Radiographic lesions and symptoms worsened; bloody sputum was also noted. Four weeks after this CT scan was taken, M. abscessus subspecies abscessus, instead of M. intracellulare, was isolated. $\mathbf{d}$ Chest CT scan at 6 months after initial isolation of $M$. abscessus subspecies abscessus. The patient's symptoms continued to worsen and sputum persistently tested positive for M. abscessus subspecies abscessus

species, alternative isolation of two or three species, or simultaneous isolation of multiple species in same patients [11].

We have experienced several patients with NTM lung diseases in whom changes of NTM species was identified during or after treatment. However, the clinical significance this of NTM change has not yet been reported. Therefore, the aim of this study was to determine the clinical implications of changes of NTM species during or after treatment completion in patients with NTM lung disease.

\section{Methods}

\section{Study population}

Among patients treated for NTM lung disease at Seoul National University Hospital between January 1, 2009 and December 31, 2015, patients with changes of NTM species during treatment or within 2 years of treatment completion were included in the analysis. Demographic, clinical, microbiological, and radiographic data of the included patients were reviewed. This study was conducted in accordance with the amended Declaration of Helsinki. The study protocol was approved by the institutional review board of Seoul National University Hospital (IRB No: 1608-046-784), and written informed consent was obtained from all patients.

\section{Diagnosis of NTM lung disease and definition of newly isolated NTM species}

Patients were diagnosed with NTM lung disease based on the diagnostic criteria of the American Thoracic Society (ATS)/Infectious Diseases Society of America (IDSA) guideline. [12] "Change of NTM species" were defined as disappearance of initially isolated NTM species and isolation of new species at least 2 times.

\section{Follow up and treatment}

Patients with NTM lung disease underwent follow-ups every 3 to 6 months and treatment was offered in cases of significant radiographic progression (i.e. new cavity formation) or worsening respiratory symptoms (i.e. development of hemoptysis). Once treatment was initiated, patients visited the clinic every 4 to 8 weeks for physical examination, mycobacterial cultures of sputum, and radiographic evaluations. Treatment regimen was selected based on the ATS/IDSA guideline. [12] After treatment completion, patients underwent follow-ups every 3-6 months.

\section{Clinical and radiographic examination}

Changes in respiratory symptoms were evaluated by the on-duty physician on every visit. If patients reported increased sputum production, worsened dyspnea, or 
development of hemoptysis, the symptoms were regarded as "worsened". Likewise, "no change" of symptoms and "improved" symptoms were defined based on patients' report.

Chest computed tomography (CT) was performed every 6 months during treatment and every 1-2 years after treatment completion. The severity of NTM lung diseases on CT was evaluated using a scoring system modified from a previously published one [13] by a board-certified radiologist (Fig. 1). The scoring system consists of severity, extent, and mucus plugging of bronchiectasis; severity and extent of cellular bronchiolitis; diameter, wall thickness, and extent of the cavity; nodules; and consolidation [13].

\section{Microbiological examination}

At every visit, patients were requested to submit sputum samples for mycobacterial culture. Sputum were decontaminated with same volume of $4 \%$ sodium hydroxide $(\mathrm{NaOH})$, homogenized, and concentrated by centrifugation at $3000 \times g$ for $20 \mathrm{~min}$. The processed sediments were stained using the Ziehl-Neelsen method. [12] Concentrated specimen were cultured in 3\% Ogawa medium to minimize possibility of contamination and observed weekly for 9 weeks after inoculation. Once cultured, $M$. tuberculosis and NTM were differentiated using GenProbe ${ }^{\bullet}$ method (Gen-Probe; San diego, CA, USA). [14] Following isolation of a suspected mycobacterial species, NTM was confirmed by analyzing the sequences of three genes: $16 S$ rRNA, rpoB, and tuf.

Antimycobacterial drug susceptibility tests were conducted at the Korean Institute of Tuberculosis by using broth microdilution. Minimum inhibitory concentrations (MICs) of antibiotics (amikacin, cefoxitin, ciprofloxacin, clarithromycin, imipenem, moxifloxacin, rifampicin, ethambutol, linezolid for MAC; and amikacin, cefoxitin, ciprofloxacin, clarithromycin, imipenem, moxifloxacin, and linezolid for $M$. abscessus complex) were determined according to the CLSI guidelines. [15] For MAC, isolates were considered as resistant if the MIC of clarithromycin was $\geq 32 \mu \mathrm{g} / \mathrm{ml}$ and as susceptible if the MIC of clarithromycin was $\leq 8 \mu \mathrm{g} / \mathrm{ml}$. For $M$. abscessus complex, isolates were considered as resistant if the MIC of clarithromycin was $\geq 8 \mu \mathrm{g} / \mathrm{ml}$ and as susceptible if the MIC of clarithromycin was $\leq 2 \mu \mathrm{g} / \mathrm{ml}$. Inducible resistance was considered if the MIC of clarithromycin was $\leq 2 \mu \mathrm{g} / \mathrm{ml}$ for 3 days and $\geq 8 \mu \mathrm{g} / \mathrm{ml}$ for 14 days.

\section{Statistical analysis}

Data were summarized as medians with interquartile range (IQR) with non-normal distribution. We used repeated-measures data analysis with a Friedman model to test the significance of differences in CT scores. All statistical analyses were carried out using SPSS Statistics version 20 (IBM Corp, Chicago, IL, USA) and a $P$ value $<.05$ was regarded as statistically significant.

\section{Results}

\section{Characteristics of patients}

During the study period, a total of 473 patients with NTM lung disease were diagnosed at Seoul National University Hospital. Of those, 164 patients started treatment for NTM lung disease. Among 164 patients with NTM lung disease, 54 patients (39 during treatment and 15 within 2 years of treatment completion) experienced isolation of another species of NTM. Among these 54 patients, 16 satisfied the definition of "change of NTM species", 12 patients during treatment and in 4 patients within 2 years of treatment completion. The median number of isolation of new NTM species was 5.5 (IQR 3-7). The median age of these 16 patients was 69 years (IQR 61.7-73.5) and 12 (75\%) were female. The median body mass index was 20.8 (IQR 19.2-21.4) (Table 1).

Table 1 Baseline characteristics of 16 patients with nontuberculous mycobacteria lung disease included for the analysis

\begin{tabular}{ll}
\hline Patients, No. & $N=16$ \\
\hline Age, years, median (IQR) & $69(61.7-73.5)$ \\
Sex, female & $12(75.0 \%)$ \\
BMl, kg/m², median (IQR) & $20.8(19.2-21.4)$ \\
Never smoker & $14(87.5 \%)$ \\
History of tuberculosis & $6(37.5 \%)$ \\
Underlying disease & \\
Connective tissue disease & $4(25.0 \%)$ \\
Diabetes & $3(18.7 \%)$ \\
Malignancy & $1(6.0 \%)$ \\
Respiratory symptoms & \\
Sputum & $16(100 \%)$ \\
Cough & $13(81.2 \%)$ \\
Dyspnea & $4(25.0 \%)$ \\
Hemoptysis & $3(18.7 \%)$ \\
General symptoms & \\
Weight loss & $2(12.5 \%)$ \\
Night sweating & $1(6.2 \%)$ \\
Laboratory findings, median (IQR) & \\
Leukocytes $\left(\times 10^{3} / \mu l\right)$ & $6880(5070-7542)$ \\
Hemoglobin (g/dl) & $12.6(11.9-13.3)$ \\
Cholesterol & $168(149-187.7)$ \\
Creatinine & $4(3.8-4.4)$ \\
\hline
\end{tabular}

$B M I$ body mass index, IQR interquartile range 


\section{Changes of NTM species during or after treatment for NTM lung disease}

Of 16 patients who showed change of NTM species during or after initial NTM lung disease treatment, 7 patients (43.8\%) showed changes from MAC to MAA, 4 during treatment and 3 after treatment for MAC. Five patients (31.2\%) displayed changes from M. abscessus subspecies massiliense (MAM) to MAC during treatment for MAM. The other four patients exhibited change from and to other NTM species, 3 during treatment and 1 after treatment for initial NTM (Table 2). Among 12 patients with change of NTM species during treatment, the median interval from starting treatment for initial NTM lung disease to new species isolation was 7.3 months (IQR 4.2-17.4). The median interval from treatment completion for initial NTM lung disease to isolation of a new species was median 15.6 months (IQR 14.9-16.4) among the other four patients.

\section{Changes of radiographic severities throughout treatment} of initial NTM lung diseases and isolation of new NTM Overall, the CT scores throughout the treatment course did not change. The median total CT scores at initiation of treatment for initial NTM, at 6-12 months after treatment, at isolation of a new NTM species, and at 612 months after isolation of a new NTM species was $13.2,9.6,12.9$, and 13.0 , respectively $(P=.794)$ Likewise, CT scores before and after change of NTM species did not differ (median 12.9 vs $13.0, P=.763$ ) (Table 3 ).

Table 2 Changes of NTM Species During and After Treatment

\begin{tabular}{|c|c|}
\hline & $N=16$ \\
\hline $\begin{array}{l}\text { M. avium complex } \rightarrow \text { M. } \\
\text { abscessus subspecies abscessus }\end{array}$ & $\begin{array}{l}7 \\
(43.8 \%)\end{array}$ \\
\hline $\begin{array}{l}\text { M. intracellulare } \rightarrow M \text {. } \\
\text { abscessus subspecies abscessus }\end{array}$ & 4 \\
\hline $\begin{array}{l}\text { M. avium } \rightarrow \text { M. } \\
\text { abscessus subspecies abscessus }\end{array}$ & 3 \\
\hline $\begin{array}{l}\text { M. abscessus subspecies massiliense } \rightarrow M \text {. } \\
\text { avium complex }\end{array}$ & $\begin{array}{l}5 \\
(31.2 \%)\end{array}$ \\
\hline $\begin{array}{l}\text { M. abscessus subspecies massiliense } \rightarrow M \text {. } \\
\text { avium }\end{array}$ & 3 \\
\hline $\begin{array}{l}\text { M. abscessus subspecies massiliense } \rightarrow M \text {. } \\
\text { intracellulare }\end{array}$ & 2 \\
\hline \multicolumn{2}{|l|}{ Others } \\
\hline $\begin{array}{l}\text { M. avium } \rightarrow M \text {. } \\
\text { fortuitum }\end{array}$ & $1(6.2 \%)$ \\
\hline M. avium $\rightarrow$ M. intracellulare & $1(6.2 \%)$ \\
\hline M. intracellulare $\rightarrow M$. chimera & $1(6.2 \%)$ \\
\hline $\begin{array}{l}\text { M. abscessus subspecies massiliense } \rightarrow \text { M. abscessus } \\
\text { subspecies abscessus }\end{array}$ & $1(6.2 \%)$ \\
\hline
\end{tabular}

Table 3 Change of CT Scores Throughout Treatment of Initial NTM Lung Disease and Isolation of New NTM

\begin{tabular}{|c|c|c|c|c|c|}
\hline & $\begin{array}{l}\text { At } \\
\text { initiation } \\
\text { of } \\
\text { treatment }\end{array}$ & $\begin{array}{l}\text { At 6- } \\
12 \text { months } \\
\text { after } \\
\text { treatment }\end{array}$ & $\begin{array}{l}\text { At } \\
\text { isolation } \\
\text { of new } \\
\text { NTM }\end{array}$ & $\begin{array}{l}\text { At } 6- \\
12 \text { months } \\
\text { after isolation } \\
\text { of new NTM }\end{array}$ & $\begin{array}{l}P \\
\text { Value }\end{array}$ \\
\hline \multicolumn{6}{|l|}{ Bronchiectasis, } \\
\hline Severity & $1.7(1-2)$ & $1.8(1.2-2)$ & $2(1.2-2)$ & $1.7(1.3-2)$ & .572 \\
\hline Extent & $1(1-2)$ & $1(1-2)$ & $1(1-2)$ & $1(1-2)$ & .733 \\
\hline $\begin{array}{l}\text { Mucus } \\
\text { plugging }\end{array}$ & $1(0-1)$ & $0(0-1)$ & $1(0-1)$ & $1(0-1)$ & .875 \\
\hline \multicolumn{6}{|c|}{ Cellular bronchiolitis } \\
\hline Severity & $1.8(1.6-2.2)$ & $1.7(1.2-2)$ & $2(1.6-2)$ & $1.8(1.7-2)$ & .089 \\
\hline Extent & $3(1-3)$ & $2.5(1-3)$ & $2(1.5-3)$ & $2(1-3)$ & .112 \\
\hline \multicolumn{6}{|l|}{ Cavity } \\
\hline $\begin{array}{l}\text { Diameter } \\
(\mathrm{cm})\end{array}$ & $1(0-2)$ & $0(0-1)$ & $1(0-1)$ & $1(0-2)$ & .245 \\
\hline $\begin{array}{l}\text { Wall } \\
\text { thickness } \\
(\mathrm{mm})\end{array}$ & $2(0-2.5)$ & $0(0-2.1)$ & $2(0-2)$ & $0.5(0-2.4)$ & .978 \\
\hline Extent & $1(0-1)$ & $0(0-1)$ & $1(0-1)$ & $1(0-1)$ & 1.000 \\
\hline Nodules & $1(0-1)$ & $1(0.8-1)$ & $1(1-1.5)$ & $1(0-1)$ & .572 \\
\hline Consolidation & $0(0-1)$ & $0(0-0.3)$ & $0(0-1)$ & $1(0-1)$ & .479 \\
\hline Total CT score & $\begin{array}{l}13.2(7.2- \\
17.6)\end{array}$ & $\begin{array}{l}9.6(6.4- \\
14.4)\end{array}$ & $\begin{array}{l}12.9 \\
(9.0- \\
14.8)\end{array}$ & $13(8.0-14.6)$ & .794 \\
\hline
\end{tabular}

Data are expressed as median (IQR). CT computed tomography, NTM nontuberculous mycobacteria

\section{Changes of respiratory symptoms with isolation of new NTM species}

Six out of seven patients with change from MAC to MAA reported worsening of symptoms with isolation of new species. Conversely, no patient with change from MAM to MAC complained of worsening symptoms (Table 4).

\section{In vitro drug susceptibility to clarithromycin of newly isolated NTM species}

All MAA isolated during or after the treatment for MAC lung diseases showed inducible resistance to clarithromycin. MAA isolated during treatment for MAM lung disease and $M$. fortuitum isolated during treatment for MAC lung diseases also showed inducible resistance to clarithromycin. One out of three MAC species isolated during or after treatment for MAM showed resistance to clarithromycin (Table 4). This patient refused to use intravenous drugs and took azithromycin only.

\section{Treatment for newly isolated NTM species}

Among 12 patients in whom another NTM species were isolated during treatment for initial NTM lung disease, the same treatment regimens were continued in 4 patients while the regimens were modified for the newly 
Table 4 Symptomatic and Radiographic Changes, Clarithromycin Resistance, and Treatment for Newly Isolated NTM

\begin{tabular}{lllllll}
\hline No NTM spices & $\begin{array}{l}\text { Symptom } \\
\text { changes at } \\
\text { isolation of new }\end{array}$ & $\begin{array}{l}\text { Radiographic } \\
\text { changes at }\end{array}$ & $\begin{array}{l}\text { Clarithromycin } \\
\text { isolation of new }\end{array}$ & Clarithromycin & Timing of & Treatment for \\
NTM & NTIC, $\mu \mathrm{g} / \mathrm{ml})$ & NTM(MIC, $\mu \mathrm{m} / \mathrm{ml})$ & new NTM & isolation & newly isolated \\
& NTM & & &
\end{tabular}

M. avium complex $\rightarrow$ M. abscessus subspecies abscessus

$1 \quad M$. intracellulare $\rightarrow$ abscessus subspecies abscessus

3 $M$. intracellulare $\rightarrow M$. abscessus subspecies abscessus Wors

Worsening Unchanged Susceptible (2)

$\begin{array}{ll}\text { Inducible resistance } & \text { After } \\ (1,64) & \text { treatment } \\ & \text { for initial } \\ & \text { NTM }\end{array}$

Inducible resistance $\begin{array}{ll}(2,64) & \text { treatment } \\ \text { for initial }\end{array}$ NTM

Inducible resistance After $\begin{array}{ll}(2,8) & \text { treatment } \\ & \text { for initial }\end{array}$ NTM

$4 \quad$ M. intracellulare $\rightarrow M$. abscessus subspecies abscessus

Worsening

Susceptible (2)

Inducible resistance $(0.5,64)$

After treatment for initial NTM

$5 \quad$ M. avium $\rightarrow$ M. abscessus subspecies abscessus

Unchanged

Unchanged subspecies abscessus

$6 \quad$ M. avium $\rightarrow$ M. abscessus subspecies abscessus

Worsening

(a)

Worsening

Worsening

$7 \quad$ M. avium $\rightarrow$ M. abscessus subspecies abscessus

Unchanged

Susceptible (0.5)

$$
(1,64)
$$

Inducible resistance During $(0.5,64) \quad$ treatment for initial NTM

$\begin{array}{ll}\text { Inducible resistance } & \text { During } \\ (1,64) & \text { treatment } \\ & \text { for initial } \\ & \text { NTM }\end{array}$

Continued on-going treatment

M. abscessus subspecies massiliense

$\rightarrow$ M. avium complex

8 M. abscessus subspecies massiliense $\rightarrow M$. intracellulare

9 M. abscessus subspecies massiliense $\rightarrow M$. intracellulare

10 M. abscessus subspecies massiliense $\rightarrow$ M. avium

11 M. abscessus subspecies massiliense $\rightarrow$ M. avium

12 M. abscessus subspecies massiliense $\rightarrow M$. avium

Unchanged

Unchanged

N/A

N/A

Unchanged

Unchanged

Susceptible (0.5)

Resistance (64)

Unchanged

Improving

Susceptible (0.5)

Susceptible (2)

During treatment for initial NTM

Modified regimen and continued treatment

Not started$$
\text { treatment }
$$

$\begin{array}{ll}\text { During } & \text { Modified } \\ \text { treatment } & \text { regimen and }\end{array}$ treatment regimen and NTM treatment

Modified

During treatment for initial NTM

regimen and

continued

During treatment for initial NTM

During
treatment
for initial
NTM
During
treatment
for initial
NTM

treatment

Continued on-going treatment

Modified regimen and continued treatment

astarted NTM

Others

13 M. avium $\rightarrow$ M. fortuitum
Unchanged Improving
Susceptible (0.5)
Not started 
Table 4 Symptomatic and Radiographic Changes, Clarithromycin Resistance, and Treatment for Newly Isolated NTM (Continued)

\begin{tabular}{|c|c|c|c|c|c|c|c|}
\hline No & NTM spices & $\begin{array}{l}\text { Symptom } \\
\text { changes at } \\
\text { isolation of new } \\
\text { NTM }\end{array}$ & $\begin{array}{l}\text { Radiographic } \\
\text { changes at } \\
\text { isolation of new } \\
\text { NTM }\end{array}$ & $\begin{array}{l}\text { Clarithromycin } \\
\text { resistance for initial } \\
\text { NTM }(M I C, \mu g / m l)\end{array}$ & $\begin{array}{l}\text { Clarithromycin } \\
\text { resistance for new } \\
\text { NTM(MIC, } \mu \mathrm{g} / \mathrm{ml})\end{array}$ & $\begin{array}{l}\text { Timing of } \\
\text { new NTM } \\
\text { isolation }\end{array}$ & $\begin{array}{l}\text { Treatment for } \\
\text { newly isolated } \\
\text { NTM }\end{array}$ \\
\hline 14 & M. avium $\rightarrow$ M. intracellulare & Worsening & Worsening & $\mathrm{N} / \mathrm{A}$ & Susceptible (1) & $\begin{array}{l}\text { After } \\
\text { treatment } \\
\text { for initial } \\
\text { NTM }\end{array}$ & Started \\
\hline 15 & $\begin{array}{l}\text { M. intracellulare } \rightarrow M \text {. } \\
\text { chimerae }\end{array}$ & Improving & Worsening & Susceptible (1) & Susceptible (2) & $\begin{array}{l}\text { During } \\
\text { treatment } \\
\text { for initial } \\
\text { NTM }\end{array}$ & $\begin{array}{l}\text { Continued } \\
\text { on-going } \\
\text { treatment }\end{array}$ \\
\hline 16 & $\begin{array}{l}\text { M. abscessus subspecies } \\
\text { massiliense } \rightarrow \text { M. abscessus } \\
\text { subspecies abscessus }\end{array}$ & Unchanged & Worsening & Susceptible (0.5) & $\begin{array}{l}\text { Inducible resistance } \\
(1,16)\end{array}$ & $\begin{array}{l}\text { During } \\
\text { treatment } \\
\text { for initial } \\
\text { NTM }\end{array}$ & $\begin{array}{l}\text { Continued } \\
\text { on-going } \\
\text { treatment }\end{array}$ \\
\hline
\end{tabular}

MIC minimal inhibitory concentration, N/A not available, NTM nontuberculous mycobacteria

${ }^{a}$ Treatment for initial NTM lung disease was completed. Then, treatment for newly isolated NTM was initiated

isolated NTM in 4 patients. Despite of isolation of new NTM species, the other 4 patients continued to receive and completed treatments for initial NTM lung disease and were observed for a while; in 2 out of these patients, treatments for newly isolated NTM were started eventually. (Table 4).

Among 4 patients in whom new NTM species were isolated after the completion of treatment for initial NTM lung disease, only one started to receive treatment for newly isolated NTM. (Table 4).

\section{Discussion}

In this study on patients with NTM lung diseases who received treatment, we identified several interesting findings. First, approximately $10 \%$ of patients with NTM lung disease exhibited changes to new NTM species during or within 2 years after treatment completion. Second, the most common pattern of NTM change species was from MAC to MAA. Third, change from MAC to MAA was associated with worsening of respiratory symptoms and radiographic lesions and most importantly with inducible resistance of clarithromycin.

Simultaneous or sequential isolation of several NTM species in the same patients has been reported. [10, 11, 16] In addition, repeated culture of different MAC strains in the same among patients with nodular bronchiectatic lung disease was also identified. [16] Through this study, we identified conversion of NTM species during or after treatment in 16 (9.8\%) out of 164 patients treated for NTM lung diseases. The Majority of these patients (11 out of 16) experienced symptomatic and/or radiographic worsening with isolation of new NTM species. Similarly to the recent study [17], these could be regarded as development of new NTM lung disease.

In our study, conversion from lung disease by MAC to the caused by MAA, which is more difficult to treat, was most common than other conversion patterns.
Furthermore, all MAA isolated from patients who had been treated for MAC lung disease showed inducible resistance to clarithromycin. Inducible resistance to clarithromycin involves a functional erm(41) gene, related to a T/C polymorphism at the 28th nucleotide. $[18,19]$ The rate of inducible resistance among these patients was very high compared with our recent study, which showed $55.1 \%$ of MAA isolated from patients in our hospital. [20] It could be explained that MAA with C28 sequevar, which do not show inducible resistance, might be eradicated by previous treatment with regimen including macrolide.

Previous studies reported that patients with different MAC strains usually have nodular bronchiectatic features rather than cavitary disease. $[16,21]$ Different strains might reside in different ectatic bronchi or nodules in same patients. Likewise, in our study, all 16 patients with conversion of NTM species during or after treatment had nodular bronchiectatic features. We speculate that these patients already had two NTM species in different ectatic bronchi or nodules at initial diagnosis of NTM lung disease. The number of initially detected major NTM species might be minimized with treatment while minor NTM species prevailed. For patients with conversion from MAC lung disease to MAA lung disease, treatment with macrolide, ethambutol, and rifampicin might reduce the number of MAC as well as MAA which is sensitive to clarithromycin, but MAA with inducible resistance could resist treatment and turn into the major NTM species in those patients.

This study has several limitations. First, the number of patients included in the analysis was small, although all patients included were treated at an institution that routinely diagnoses and treats a large number patient with NTM lung disease. To confirm our observations, a large-scale study enrolling patients from multiple hospitals is needed. Secondly, this study was performed retrospectively. Time points of requesting mycobacterial 
culture of sputum or chest CT scans were not controlled strictly. Additionally, crucial data, such as drug susceptibility test for the secondary isolated NTM species, were missing in some patients.

\section{Conclusions}

NTM species changes could occur during or after treatment for NTM lung disease. Especially, changes from MAC to MAA were accompanied by symptomatic and radiographic worsening as well as inducible resistance to clarithromycin.

\section{Abbreviations}

CT: Chest computed tomography; IQR: Interquartile range; MAA: M. abscessus subspecies abscessus; MAC: M. avium complex; MAM: M. abscessus subspecies massiliense; MICs: Minimum inhibitory concentrations; NTM: nontuberculous mycobacteria

\section{Funding}

There was no funding for this study.

\section{Availability of data and materials}

The datasets used and/or analysed during the current study available from the corresponding author on reasonable request.

\begin{abstract}
Authors' contributions
JSL contributed to the data acquisition, data interpretation, and writing of the manuscript. JHL, SHY, contributed to the collection and analysis of radiological data. TSK, HWS, SKH contributed substantially to the study design, data analysis and interpretation, and the writing of the manuscript. $J J Y$ is the guarantor of the entire manuscript and is responsible for the content of the manuscript, including the data collected and its analysis. All authors read and approved the final manuscript.
\end{abstract}

\section{Ethics approval and consent to participate}

The study protocol was approved by the Institutional Review Board of Seoul National University Hospital (IRB No: 1608-046-784) and was conducted in accordance with the amended Declaration of Helsinki. All participants provided written informed consent before enrollment.

\section{Consent for publication}

Not applicable

\section{Competing interests}

The authors declare that they have no competing interests.

\section{Publisher's Note}

Springer Nature remains neutral with regard to jurisdictional claims in published maps and institutional affiliations.

\section{Author details}

${ }^{1}$ Division of Pulmonary and Critical Care Medicine, Department of Internal Medicine, Seoul National University College of Medicine, 101, Daehak-ro, Jongno-gu, Seoul 03080, Republic of Korea. ${ }^{2}$ Department of Radiology, Seoul National University College of Medicine, 101, Daehak-ro, Jongno-gu, Seoul 03080, Republic of Korea. ${ }^{3}$ Department of Laboratory Medicine, Seoul National University Hospital, 101, Daehak-ro, Jongno-gu, Seoul 03080, Republic of Korea.

Received: 10 July 2017 Accepted: 29 November 2017

Published online: 20 December 2017

\section{References}

1. Prevots DR, Marras TK. Epidemiology of human pulmonary infection with nontuberculous mycobacteria: a review. Clin Chest Med. 2015;36(1):13-34.

2. Falkinham JO. Environmental sources of nontuberculous mycobacteria. Clin Chest Med. 2015;36(1):35-41.
3. Tortoli E. Microbiological features and clinical relevance of new species of the genus mycobacterium. Clin Microbiol Rev. 2014:27(4):727-52.

4. Tortoli E. The new mycobacteria: an update. FEMS Immunology \& Medical Microbiology. 2006:48(2):159-78.

5. Stout JE, Koh W-J, Yew WW. Update on pulmonary disease due to nontuberculous mycobacteria. Int J Infect Dis. 2016;45:123-34.

6. Park Y, Lee C, Lee S, Yang S, Yoo C, Kim Y, Han S, Shim Y, Yim J. Rapid increase of non-tuberculous mycobacterial lung diseases at a tertiary referral hospital in South Korea [short communication]. The International Journal of Tuberculosis and Lung Disease. 2010;14(8):1069-71.

7. Lee SK, Lee EJ, Kim SK, Chang J, Jeong SH, Kang YA. Changing epidemiology of nontuberculous mycobacterial lung disease in South Korea. Scand J Infect Dis. 2012;44(10):733-8,

8. Koh W-J, Kwon OJ, Jeon K, Kim TS, Lee KS, Park YK, Bai GH. Clinical significance of nontuberculous mycobacteria isolated from respiratory specimens in Korea. CHEST Journal. 2006;129(2):341-8.

9. Jang M-A, Koh W-J, Huh HJ, Kim S-Y, Jeon K, Ki C-S, Lee NY. Distribution of nontuberculous mycobacteria by multigene sequence-based typing and clinical significance of isolated strains. J Clin Microbiol. 2014;52(4):1207-12.

10. Griffith DE, Girard WM, Wallace Jr RJ. Clinical features of pulmonary disease caused by rapidly growing mycobacteria: an analysis of 154 patients. Am Rev Respir Dis. 1993;147(5):1271-8.

11. Lim H-J, Park CM, Park YS, Lee J, Lee S-M, Yang S-C, Yoo C-G, Kim YW, Han SK, Yim J-J. Isolation of multiple nontuberculous mycobacteria species in the same patients. Int J Infect Dis. 2011;15(11):e795-8.

12. Griffith DE, Aksamit T, Brown-Elliott BA, Catanzaro A, Daley C, Gordin F, Holland SM, Horsburgh R, Huitt G, lademarco MF. An official ATS/IDSA statement: diagnosis, treatment, and prevention of nontuberculous mycobacterial diseases. Am J Respir Crit Care Med. 2007;175(4):367-416.

13. Kim HS, Lee KS, Koh W-J, Jeon K, Lee EJ, Kang H, Ahn J. Serial CT findings of Mycobacterium Massiliense pulmonary disease compared with mycobacterium abscessus disease after treatment with antibiotic therapy. Radiology. 2012;263(1):260-70.

14. Bergmann JS, Yuoh G, Fish G, Woods GL. Clinical evaluation of the enhanced gen-probe amplified mycobacterium tuberculosis direct test for rapid diagnosis of tuberculosis in prison inmates. J Clin Microbiol. 1999; 37(5):1419-25.

15. CLSI: Susceptibility testing of Mycobacteria, Nocardiae, and other aerobic Actinomycetes. Approved standard-second edition CLSI document M24-A2 Wayne, PA: clinical and laboratory standards institute 2011.

16. Wallace Jr RJ, Zhang Y, Brown BA, Dawson D, Murphy DT, Wilson R, Griffith DE. Polyclonal Mycobacterium Avium Complex infections in patients with nodular bronchiectasis. Am J Respir Crit Care Med. 1998;158(4):1235-44.

17. Griffith DE, Philley JV, Brown-Elliott BA, Benwill JL, Shepherd S, York D, Wallace RJ. The significance of mycobacterium abscessus subspecies abscessus isolation during Mycobacterium Avium Complex lung disease therapy. CHEST Journal. 2015;147(5):1369-75.

18. Nash KA, Brown-Elliott BA, Wallace RJ. A novel gene, erm (41), confers inducible macrolide resistance to clinical isolates of mycobacterium abscessus but is absent from mycobacterium chelonae. Antimicrob Agents Chemother. 2009;53(4):1367-76

19. Choi H, Kim S-Y, Kim DH, Huh HJ, Ki C-S, Lee NY, Lee S-H, Shin S, Shin SJ, Daley CL. Clinical characteristics and treatment outcomes of patients with acquired Macrolide-resistant mycobacterium abscessus lung disease. Antimicrob Agents Chemother. 2017;61(10):e01146-17.

20. Park J, Cho J, Lee CH, Han SK, Yim JJ. Progression and treatment outcomes of lung disease caused by mycobacterium abscessus and Mycobacterium Massiliense. Clin Infect Dis. 2017;64(3):301-8.

21. Mazurek GH, Hartman S, Zhang Y-s, Brown B, Hector J, Murphy D, Wallace R, Large DNA. Restriction fragment polymorphism in the Mycobacterium Avium-M. Intracellulare complex: a potential epidemiologic tool. J Clin Microbiol. 1993;31(2):390-4. 